\title{
Heritable pulmonary arterial hypertension
}

INSERM

\section{Source}

INSERM. (1999). Orphanet: an online rare disease and orphan drug data base. Heritable pulmonary arterial hypertension. ORPHA:275777

Heritable pulmonary arterial hypertension (HPAH) is a form of pulmonary arterial hypertension (PAH, see this term), occurring due to mutations in $\mathrm{PAH}$ predisposing genes or in a familial context. HPAH is characterized by elevated pulmonary arterial resistance leading to right heart failure. HPAH is progressive and potentially fatal. 